QUALITATIVE RESEARCH ARTICLE

\title{
Qualitative Thematic Analysis of Pharmacy Student Blogs for International Advanced Pharmacy Practice Experiences
}

\author{
Stephanie Coghill, PharmD, ${ }^{\mathrm{a}, \mathrm{b}}$ Valerie D Chaffee, PharmD, ${ }^{\mathrm{c}, \mathrm{d}}$ Melody Ryan, PharmD, MPH ${ }^{\mathrm{b}}$ \\ ${ }^{\text {a }}$ Advocate Lutheran General Hospital \\ ${ }^{\mathrm{b}}$ University of Kentucky, College of Pharmacy \\ ${ }^{\mathrm{c}}$ Cancer and Hematology Centers of Western Michigan \\ ${ }^{\mathrm{d}}$ University of Kentucky Healthcare
}

Corresponding Author: Melody Ryan, University of Kentucky, College of Pharmacy, 725 Rose St., Lexington, KY 40536-0082. Tel: 859-257-8790. Email: melody.ryan@uky.edu

Submitted April 2, 2021; accepted October 8, 2021; ePublished October 2021

Objective. The primary objective of this study is to gain an understanding of student pharmacist learning occurring during international advanced pharmacy practice experiences (APPEs). The secondary objective is to direct the development of suitable pre-departure orientation activities for pharmacy students.

Methods. University of Kentucky College of Pharmacy students authored blogs between 2012 and 2019 describing patient-care and non-patient care international APPEs. Investigators utilized inductive thematic analysis to analyze the blogs and define codes, categories, and themes from the data set. The entire data set was utilized to generate changes for pre-departure orientation.

Results. The analysis included 47 blogs from which seven pictorial codes and 24 text codes were isolated from the data set to ultimately form four categories: Learning About Health care; My Surroundings; Logistics; and Me, Myself, and I. Two overall themes emerged from the codes and categories: Everything is Different and Here's What I Think and Feel. Examination of the data in conjunction with review of other studies focusing on study abroad demonstrated that the themes isolated in this study parallel previously described benefits of study abroad. Additionally, analysis of the blogs suggested expansion of pre-departure orientation for students and follow-up discussion to ensure understanding may be required prior to travel.

Conclusion: This study gives a unique insight into thoughts and relative importance of pharmacy student experiences during study abroad. Evaluation of the data and review of previously published studies will guide educators in refining pre-departure material. Further studies are needed to evaluate the effectiveness of revised pre-departure orientation. Keywords: international, advanced pharmacy practice experiences, blog, qualitative research, global health

\section{INTRODUCTION}

The health care industry is increasingly globalized as clinicians search for answers to the complex problems of disease state management and health care infrastructure. ${ }^{1,2}$ In order to gain a global perspective of health care, many student pharmacists are electing to participate in international experiences. ${ }^{3}$ Pharmacy schools offer a variety of international experiences ranging from advanced pharmacy practice experiences (APPEs) to globally focused courses that provide exposure to diverse cultures and health care practices. ${ }^{4}$

Students who participate in international experiences often recognize their experiences are impactful, but may be unable to articulate exactly what skills, knowledge, and attitudes they gleaned. It is useful to understand what learning occurs on international APPEs. Previous studies completed within other health care professions have found cultural immersion, cultural awareness, cultural competence, personal and professional growth, and ability to work in resourcelimited settings are positive experiences of students participating in study abroad. ${ }^{5-8}$ While some of these findings may be applicable to pharmacy students, there are cultural differences between the professions such that it is preferable to not assume that what applies to another profession's students is also true for student pharmacists. ${ }^{9,10}$ Only one study has examined the impact of international APPEs on pharmacy students. ${ }^{11}$ Steeb, et al. used a mixed-methods, longitudinal study to evaluate pharmacy students completing an international APPE using a retrospective pre/post survey to assess students' self-perceived growth across 13 competencies established by the Consortium of Universities for Global Health (CUGH) and focus group interviews. The authors sought to determine the effect of country income classification and duration of experience on learning outcomes. Their analysis found learning outcomes differed between students who went to a low- to middle-income country compared to students who went to a high-income country, noting both locations 
provided professional and personal development. ${ }^{11}$ However, the Steeb, et al. study was retrospective and asked specific questions, potentially missing learning that students did not deem pertinent. Additional information about the unique value a global experience adds to pharmacy education is needed.

The expansion of international opportunities for pharmacy students requires concurrent assessment of the preparation of these students for their experiences. A majority of studies concerning preparation for global experiences focus on medical and nursing students, with emphasis on cross-cultural content and student health and safety while abroad. ${ }^{12-16}$ The Working Group of Ethics Guidelines for Global Health Training (WEIGHT) guidelines detail best practices for global health experiences and include suggestions for pre-departure orientation. ${ }^{17}$ The WEIGHT guidelines have specific recommendations for host and sending institutions regarding formal pre-departure orientation for trainees and mentors, including: standards of practice; cultural competence; conflict resolution; language capability; and personal safety. However, students often either do not receive pre-departure preparative orientation or they feel their orientation underprepared them for challenges such as ethical considerations or poor patient outcomes. ${ }^{18}$ The American Association of Colleges of Pharmacy Global Pharmacy Education Special Interest Group created the only document specific to pharmacy student preparation. ${ }^{19}$ It includes general considerations for student preparation: host country description, communication, passport/visa requirements, airline travel, safety, housing, in-country transportation, health-related issues, travel alerts/warnings, and finances. ${ }^{19}$ Ensuring pharmacy students are adequately prepared for their international experiences is essential to maximize learning and avoiding "medical voluntourism". ${ }^{20}$ Further, it is important to know if the pre-departure orientation provided matches the issues encountered during international experiences.

Students at the University of Kentucky College of Pharmacy (UKCOP) completing international APPEs participate in a pre-departure discussion about travel logistics, communication, health, safety, cultural respect, preceptor interactions, evaluation, privacy, ethics, and professionalism. However, a formal assessment has not been conducted to determine if preparation is adequate.

The primary objective of this study is to understand the types of learning taking place on international APPEs. The secondary objective is to further direct the development of suitable pre-departure orientation activities for pharmacy students. Utilizing first-hand, in-the-moment accounts from pharmacy students' experiences, this study specifically examined students' impressions of their learning experiences and their perceptions of preparedness.

\section{METHODS}

This study utilized an inductive thematic analysis approach, where qualitative data was categorized without attempting to match the researchers' assumptions, in order to find a repeated pattern of meaning (ie, a theme). ${ }^{21}$ This approach, while time-consuming, is comprehensive and was chosen to allow the data itself to inform the findings and to avoid missing crucial insights (unknown unknowns) imbedded in the blogs. In this type of study, codes, categories, and themes are generated. Codes are individual sections of the data which "are interesting to the analyst". ${ }^{21}$ Categories, sometimes called sub-themes, are groupings of codes that support a common idea. A theme is an overarching group of categories that relate back to the initial research question. ${ }^{21}$

The authors' relationship to the research is described to fully disclose the relationship to subjects as well as the research questions. One author (MR) is the Director for International Professional Student Education and responsibilities include: development and maintenance of all international APPEs, selection of students to participate in international APPEs alongside a committee, and provision of pre-departure orientation and post-experience debriefing for all students participating. Two authors (SC and VDC) participated in international APPEs as students, one at the University of Kentucky and one at another institution.

Students completing their final, experiential year of education with a grade point average of at least 3.0 on a 4.0 scale were eligible to participate in international APPEs. Students apply for the experiences during their third year and rotation times occur throughout the experiential fourth year. During pre-departure orientation, students are instructed to communicate with UKCOP at least weekly; a blog is presented as one option to fulfill this requirement. Students are instructed to write about anything they like on their blogs with the caveat that the blogs are in the public domain and might be widely read. No prompts for the blog posts are given. At sites where two or more students are placed together, some groups elect to keep one blog and others keep separate blogs. Therefore, the unit of analysis was the blog, rather than the student. Any student who was uncomfortable with posting to a blog could elect to communicate with the UKCOP via email on a weekly basis.

Publicly available pharmacy student blogs authored between 2012 and 2019 describing international APPEs compromised the data set. All blogs were included. The Institutional Review Board determined that review was not needed to evaluate student perspectives through retrospective review because students determined the content of the blogs and the blogs are in the public domain.

During the initial blog analysis, the three investigators independently read the blogs in their entirety and began to 
generate a list of codes. Codes were generated for both the text (text codes) and for the photos (photo codes) that the students posted. The investigators met following the first review of the blogs to define a common initial list of codes. Each investigator then re-read the blogs and coded data, such as photos or sentences, that fit the predetermined list. Following the second blog analysis, the investigators met again to resolve any discrepancies. The final resultant data set was an agreed upon list of codes (Table 1).

Once the codes were finalized, investigators independently grouped codes to generate a list of potential categories. Subsequent meetings of the investigators aligned the initial coded data set into final categories and overall themes. Once compiled, the final themes and categories were used by the authors to reflect upon the pre-departure preparation methods.

\section{RESULTS}

Nine APPE sites across seven countries were represented in 47 student blogs written over seven years (20122019). Twenty blogs focused on patient care elective sites: Poznan University of Medical Sciences, Poland ( $\mathrm{n}=8)$; Mahidol University, Thailand ( $n=6)$; Hombro a Hombro Health Center, Ecuador $(n=4)$; and Peking University Third Hospital, China $(n=2)$. The 27 remaining blogs focused on non-patient care electives with a research or managed care focus: National Health Services (NHS) National Services Scotland, Scotland (n=8); University of Manchester, England $(\mathrm{n}=7)$; NHS Lothian Pharmacy Service, Scotland $(\mathrm{n}=6)$; NHS Education for Scotland, Scotland ( $\mathrm{n}=3)$; and British Columbia Ministry of Health, Pharmaceutical Services Division Drug Use Optimization Branch, Canada (n=3). One blog was less than one-half of a typed page and contained no codes; it was excluded from the data set.

The investigators found seven pictorial codes and 24 text codes, for a total of 31 codes throughout 46 blogs. An average of $19.1 \pm 6$ codes (median: 19.5, range: 6-31) were found in each blog. Four categories were formed from the codes: Learning About Health care; My Surroundings; Logistics; and Me, Myself, and I. At least one code per category was noted in $83 \%(n=38)$ of the blogs. The categories Learning About Health care and Me, Myself, and I lacked code representation within two and six blogs, respectively. Figure 1 represents the frequency of each code by country for each category. The four categories were combined into two themes: Everything is Different and Here's What I Think and Feel (Table 1).

The first major theme identified in all blogs was Everything is Different, which included the categories Learning About Health care and My Surroundings. Learning About Health care gave insight to the students' experiential education during their time abroad. The most common code in this category was rotational learning $(\mathrm{n}=44)$. Other commonly discussed experiences in this category included health care differences $(n=38)$, the role of the pharmacist $(n=31)$, and medication differences $(n=25)$. A photograph of the health care setting in which the students worked was also frequently included in blog posts $(n=29)$. Patient care $(n=20)$, work conditions $(n=20)$, and health disparities and risks $(n=11)$ were less frequently cited codes in Learning About Health care. Education and training $(n=16)$, the code cited least often in this category, was usually a description of differences between the US and the host country pharmacist training.

My Surroundings, included in the Everything is Different theme, encompassed students' environment, actions, and events. Photographs showing settings that differed from home were common across all blogs, and gave rise to pictorial codes identified by the investigators such as architecture $(n=38)$, nature $(n=36)$, religion and church $(n=31)$, and local people $(n=23)$. Tourism and sightseeing $(n=44)$ was the most documented text code in this category, reflecting the students' desire to explore their host countries. Customs and culture $(n=39)$ was another recurring code. Other frequent codes in the My Surroundings category included history $(n=28)$ and current events $(n=20)$. Students frequently visited museums or attended sporting events or festivals. Language and communication $(n=33)$ issues appeared in most blogs.

The second major theme identified was Here's What I Think and Feel. Logistics, which primarily focused on the students' thoughts about external occurrences, was one of two categories that comprised this theme. Codes for accommodations and local food and drink were isolated by the investigators as both pictorial and text codes. The local food and drink text $(n=41)$ and pictorial $(n=32)$ codes were typically used to describe a diet vastly different from students' diets in the United States.

Transportation ( $\mathrm{n}=41$ ) was one of the two most frequently isolated codes in the category Logistics. Students often described alternative modes of transportation. Climate and weather $(\mathrm{n}=37)$ was commonly documented in this category. Economic differences and comparisons $(n=25)$ were a topic throughout the blogs. Some students focused on actual cost differences, such as the price of a sandwich, whereas others commented on the socioeconomic status of patients they encountered. Differences in currency were also cited. In the initial blog entry, students often noted arrangements made for their trip abroad, which was coded as preparation $(n=14)$.

Me, Myself, and I, the second category in the theme Here's What I Think and Feel, focused on the students' internal thoughts and emotions. Making friends and socializing $(\mathrm{n}=28)$ described how students felt spending time with or forming relationships with individuals they did not know prior to their international experience. Students many times 
would socialize with people they met at their rotation site itself. Travel frustrations and inconveniences $(\mathrm{n}=24)$ were a common occurrence, as students experienced problems with transportation, getting in/out of countries, or had accommodation difficulties.

Codes capturing the students' states of mind included: emotions, gratitude, and personal safety and health. Students experienced many emotions $(\mathrm{n}=19)$, for example: homesickness, excitement, culture shock, frustration, happiness, and sadness. Gratitude $(\mathrm{n}=22)$ was tabulated separately because it was documented more frequently than any other mental state. Personal safety and health $(\mathrm{n}=16)$, while less commonly documented than other codes, gave insight into the student's well-being while abroad. Some students described times while travelling that they did not feel safe. One student experienced her host country's health care system first-hand when she broke her ankle. Personal safety and health also involved students visiting health care providers in the USA pre-departure.

The entire data set was analyzed to determine effectiveness and needed changes for pre-departure orientation. Few blogs directly commented on information students were given in the pre-departure orientation. The specific codes of preparation, accommodations, personal safety and health, language and communication, transportation, customs and culture, and travel frustrations and inconveniences contained most of the information pertinent to pre-departure orientation (Table 2). Several preparation codes emerged regarding packing keeping within airline weight limits and while planning additional regional travel before, during, or after the APPE. The accommodations code demonstrated student lack of understanding of utilities such as heating, water, electricity, and appliances. Personal safety and health codes reflected upon food safety, vector-borne illnesses, and regional infectious diseases. Several students were ill or had accidents during their APPE and one group experienced a bombing in the city in which they were staying. Most blogs from the United Kingdom mentioned safety concerns from traffic driving on the left side of the road. Another concerning group of codes indicated students had difficulty finding their accommodations or communicating with landlords and dormitory personnel on arrival.

The codes for transportation and travel frustrations and inconveniences frequently demonstrated this area was neglected in student preparation. Many students had flight or other trip delays, but had never navigated these situations on their own previously. Students often expressed confusion and frustration with public transportation in the cities where they were living, frequently because they had never used these transportation systems in the United States. Despite a robust discussion of immigration and customs, several students experienced problems upon arrival in their host countries. Several students also reported scams directed at tourists while in the country.

Customs and culture was a very common code in the student blogs, indicating a great deal of learning in this area. However, some of these codes suggested additional pre-departure information that might be useful to students. Students described culturally based greetings, dress norms, table etiquette, and tipping practices that they found confusing. Related to culture, many students reported language barriers.

\section{DISCUSSION}

This study provides a unique insight to students' thoughts, emotions, and daily activities as presented in blogs kept during an international APPE. The strength of this study is that it allows for the examination of student learning recorded organically concurrent with the student experience. This is the only study in the literature that has taken this approach. Inductive thematic analysis of 47 blogs revealed students learned a great deal about international health care. Additional learning about customs, culture, and travel took place. Secondary analysis identified areas that should receive additional emphasis in pre-departure orientation: packing, accommodation orientation, health and safety, arrival plans, transportation guidelines, immigration, cultural practices, and basic language skills,

The two themes isolated in this analysis, Everything is Different and Here's What I Think and Feel, align with topics suggested for pre-departure orientation for other health care professions. Codes chronicled rotational learning, health care differences, health care settings, patient care, work conditions, and health disparities and risks and were similar to other published studies by Cox, et al. and Reardon, et al. detailing global health care rotations in nursing and medicine, such as cognitive development, ability to work in resource limited settings, and a better understanding of working within other health care systems. ${ }^{5,6}$ Certain codes were more focused on pharmacy-related learning including the role of the pharmacist, medication differences, and pharmacist education and training.

The category My Surroundings highlights codes that correlate to study abroad experiences essential to developing cultural awareness. Language and communication was a particularly interesting code in that it appeared in both Englishand non-English-speaking countries. Almost half of the blogs coded with language and communication were in countries where English was the primary language. Difficulty with language and communication has been previously noted as a stressor on international nursing students by Cox, et al.. ${ }^{5}$ Codes in My Surroundings provided insight into some of the intangible benefits of international APPEs, particularly in cultural learning. This finding is in parallel to the observation 
by Allen, et al. in which $81 \%$ of health care students demonstrated an overall increase in their cultural competency scores after an experience. ${ }^{8}$

The Logistics category was composed largely of travel essentials or reflected the student experience of being a temporary member of their host society. Transportation was one of two most cited codes within the category, which demonstrated how frequently students thought about transportation and how they were often unprepared for not having their own vehicles. Economic differences and comparisons, when mentioned in student blogs, often highlighted a learning experience regarding monetary value and socioeconomic status of the country or patients encountered.

The category Me, Myself, and I highlighted intangible experiences of education abroad, describing challenges faced and emotional reactions to experiences. Although students depicted a range of emotions, the authors noted the frequent occurrence of explicitly expressing gratitude. A change in perspective and a deeper appreciation for their home health care system and way of living was also a theme evident from interviews conducted with United Kingdom physicians-in-training by Reardon, et al. ${ }^{6}$

Overall, the findings of this study mirror major themes of study abroad experiences identified by Lu, et al. who reviewed ten studies and summarized the overall impact of global health electives on US medical residents. ${ }^{22}$ Four positive themes were identified: improvement of medical knowledge; improvement in resourcefulness and costeffectiveness; improvement in cultural and interpersonal competence; and professional and career development. ${ }^{22}$ The category Learning About Health care parallels improvement of medical knowledge, whereas the category Logistics more closely resembles improvement in resourcefulness and cost-effectiveness. Two negative themes were also identified in Lu, et al.: personal health and safety risks. ${ }^{22}$ The code Personal Safety and Health correlates to these negative themes.

The secondary goal of the study was to assist the authors in determining suitable pre-departure orientation activities. The codes identified in this study give insight into information students wished they had prior to the experience.

Currently, students are directed to guidebooks and the US Department of State website for information regarding their host country, including information on transportation, customs, health and safety, and scams. As the codes for customs and culture and personal safety and health demonstrated, students run into issues with country-specific practices, suggesting that these resources may not be utilized prior to travel. Supplementary directions for utilization of the required health and safety insurance coverage is likely needed. The codes for language and communication also demonstrated an area for improvement in pre-departure orientation. It may be prudent to request students learn a few basic phrases in the language of their country and other countries where they plan to visit and, perhaps, carry allergy and dietary information written in the local language to improve their preparedness for language barriers.

The need for further guidance on navigating both airline travel and in-country transportation systems was highlighted by the codes for transportation and travel frustrations and inconveniences. Some codes also pointed to the need for each student to have a clear plan for arrival that is not dependent on the ability to access the internet. The preparation and accommodations codes likewise suggest that students may require additional guidance in this area. Students may appreciate additional communication regarding packing for extended travel and the ability to do laundry while traveling. Students should also be reminded to ask for a thorough orientation at their accommodations, including appliances and utilities. While all of these items were separately isolated as codes in the data set, students were able to adequately problem-solve any issues encountered.

This study's findings support the need for incorporating the CUGH defined levels of global health competency into the pre-departure curriculum. The most basic level, Global Citizen, is designed to guide the development of curricula for post-secondary students interested in pursuing global health. ${ }^{26}$ Eight domains are included in the Global Citizen level: global burden of disease; globalization of health and health care; social and environmental determinants of health; collaboration, partnering, and communication; ethics; professional practice; health equity and social justice; and sociocultural and political awareness. As Steeb, et al. demonstrated, pharmacy students who participated in international APPEs in both low-middle and high-income countries experienced growth in all domains of the CUGH competencies. ${ }^{11}$ The Global Citizen-level domains are represented in this study, although CUGH competencies were not directly addressed. Adopting the CUGH Global Citizen framework as part of pre-departure learning materials for APPE students participating in international experiences may lead to an increase in student-articulated benefits of study abroad.

There were several limitations to this study. Observer bias could be present; however, this was addressed by the inclusion of three individual reviewers who adjudicated the codes, categories, and themes. Because this study was limited to blogs from student pharmacists affiliated with one institution, it may not be generalizable to other health care trainees or other institutions. Some host countries where students participated in APPEs had strict rules regarding social media; therefore, some countries have more representation than others in the data set. Cox, et al. discuss students experiencing reverse culture shock and struggling to reintegrate into society upon return to their home country. ${ }^{5}$ This study did not have the ability to assess whether or not students experienced such phenomena because the blogs often concluded prior to students' return home. 


\section{CONCLUSION}

This study gives a unique insight into pharmacy student experiences during international APPEs by using firstperson, in-the-moment accounts of the experience as the unit of analysis. In addition to rotational learning and skills essential to travel, students also gained knowledge and understanding of intangible experiences including cultural awareness, challenges faced, and emotional reactions to experiences. Gaps in pre-departure orientation were also determined, and ideas were generated to make changes to current available material. In the future, programs should focus on incorporating and evaluating key global health competencies into pre-departure materials for pharmacy students.

\section{REFERENCES}

1. Glaesser D, Kester J, Paulose H, Alizadeh A, Valentin B. Global travel patterns: an overview. Journal of Travel Medicine. 2017; 24(4):1-5.

2. Apuzzo M, Kirkpatrick D. Covid-19 changed how the world does science, together. The New York Times. April 1, 2020. Accessed July 14, 2021. https://www.nytimes.com/2020/04/01/world/europe/coronavirus-science-researchcooperation.html

3. Allard M, Raimi-Abraham B. Inclusion of pharmacy students in globalization of professional pharmacy practice. American Journal of Health-System Pharmacy. 2019; 76(1):2077-2079.

4. Steeb DR, Overman RA, Sleath BL, Joyner PU. Global experiential and didactic education opportunities at US colleges and schools of pharmacy. American Journal of Pharmaceutical Education. 2016;80(1):7.

5. Cox L, Crump L, Struwing R, Gillum D, Abraham S. Building cultural competence. Journal of Christian Nursing. 2017; 34(3):35-40.

6. Reardon C, George G, Enigbokan O. The benefits of working abroad for British General Practice trainee doctors: the London deanery out of programme experience in South Africa. BMC Medical Education. 2015; 15(1):174.

7. Kelleher, S. Perceived benefits of study abroad programs for nursing students: an integrative review. Journal of Nursing Education. 2013; 52(12):690-695.

8. Allen C, Smart D, Odom-Maryon T, Swain D. The value of community-focused interprofessional care in Peru for developing cultural competency in health professions students. International Journal of Nursing Education Scholarship. 2013; 10(1):153-162.

9. Hall P. Interprofessional teamwork: professional cultures as barriers. Journal of Interprofessional Care. $2005 ; 19$ Suppl 1:188-96.

10. Pecukonis E, Doyle O, Bliss DL. Reducing barriers to interprofessional training: promoting interprofessional cultural competence. Journal of Interprofessional Care. 2008;22(4):417-28.

11. Steeb DR, Miller ML, Schellhase EM, Malhotra JV, McLaughlin JE, Dascanio SA, Haines ST. Global health learning outcomes in pharmacy students completing international advanced pharmacy practice experiences. American Journal of Pharmaceutical Education. 2020;84(3):7586.

12. Reid M, Biller N, Lyon S, Reilly J, Merlin J, Dacso M, Friedman H. Reducing risk and enhancing education: U.S. medical students on global health electives. American Journal of Infection Control. 2014; 42(1):1319-1321.

13. Imperato P, Bruno D, Sweeney M. Ensuring the health, safety, and preparedness of U.S. medical students participating in global health electives overseas. Journal of Community Health. 2016; 41(1):442-450.

14. Dougherty A, Goller T, McNamara M, Moore M. One more thing before you go: pre-departure training for medical students on global health rotation. Lecture presented at: International Association of Medical Science Educators Conference; June 10-13, 2017; Burlington, VT.

15. Bentley R, Ellison K. Increasing cultural competence in nursing through international service-learning experiences. Nurse Educator. 2007; 32(5):207-211.

16. Anderson K, Slatnik M, Pereira I, Cheung E, Xu K, Brewer T. Are we there yet? Preparing Canadian medical students for global health electives. Academic Medicine: Journal of the Association of American Medical Colleges. 2012; 87(2):206-209.

17. Crump J, Sugarman J, Working Groups on Ethics Guidelines for Global Health Training (WEIGHT). Ethics and best practice guidelines for training experiences in global health. The American Journal of Tropical Medicine and Hygiene. 2010;83(6):1178-1182.

18. Kironji A, Aluri J, Decamp M, et al. Gaps in predeparture training and postexperience briefing in global health experiences: a survey of health professions students. Annals of Global Health. 2014; 80(3):166-167.

19. Alsharif N, Dakkuri A, Abrons J, Williams D, Ombengi D, Zheng H, Al-Dahir S, Tofade T, Gim S, O'Connell M, Ratka A, Dornblaser E. Current practices in global/international advanced pharmacy practice experiences: 
home/host country or site/institution considerations. American Journal of Pharmaceutical Education. 2016; 80(3):38.

20. McLennan S. Medical voluntourism in Honduras: 'helping' the poor? Progress in Development Studies. 2014; 14(2):163-179.

21. Braun V, Clarke V. Using thematic analysis in psychology. Qualitative Research in Psychology. 2006; 3(2):77101.

22. Lu P, Park E, Rabin T, et al. Impact of global health electives on US medical residents: a systematic review. Annals of Global Health. 2018; 84(4): 692-703.

23. Jogerst $\mathrm{K}$, Callender B, Adams V, et al. Identifying interprofessional global health competencies for 21 st-century health professionals. Annals of Global Health. 2015; 81(2): 239-247. 
Table 1. Frequency of Code Occurrence Isolated in Pharmacy Student Blogs and Example Student Quotes Supporting Themes, Categories, and Codes

\begin{tabular}{lll}
\multicolumn{1}{c}{ Theme } & \multicolumn{1}{c}{ Category } & \multicolumn{1}{c}{ Code } \\
\hline Everything & Learning & Rotational Learning \\
Is Different & About &
\end{tabular}

Healthcare

HIV. The review was very comprehensive and we got to discuss treatment, preexposure prophylaxis, and post-exposure prophylaxis.

Healthcare Differences

38 (82.6) The Thai healthcare and health insurance system is very different from the U.S. It is a national system with three separate entities. I will try my best to give you a short explanation, in hopes that I don't over simplify them because of my brevity. The first form of coverage is for civil servants (or government employees), their coverage is the best of all three but they are also paid lower wages than most citizens. They are able to go to any hospital they choose, whether it is private or public. They also have free access to drugs on the NLED, National List of Essential Drugs, and some medications that are not on the NLED. The second form of coverage is called Universal coverage, these patients have a choice of a pre-approved hospital they register at. The hospitals they can pick from are mostly public but it varies according to the province in which the patient lives. They also only have free access to medications on the NLED. The final form of coverage is Social Security Scheme.

These patients also get their choice of hospital but have both public and private options, unlike the UC patients. They also have access to medications on the NLED plus some additional medication coverage. The BIG difference in Thai health care is that you chose your hospital and then you CANNOT change, so the small variance in plans make a big difference because the hospital is where all your paid care comes from, whether it is primary care (generally outpatient) or tertiary care (inpatient care).

The Role of the Pharmacist

Healthcare Setting (photo) Medication Differences

Patient Care

Work Conditions

Education and Training
$31(67.4)$ Pharmacists are able to diagnose when patients arrive at the pharmacy and then can assist with antibiotic selection and dispensing. Even diabetic, hypertension, and asthma medications are available to be prescribed by the pharmacist.

$29(63.0)$

25 (54.3) Long-acting insulins are not offered in BC. Rabeprazole is the PPI of choice. Ramipril is the ACEI of choice. Clopidogrel requires prior authorization.

22 (47.8) [We] see all new patients on all four Medicine of the Elderly wards each day and make a patient profile for them. We document all of the errors we find and make note of any clinical issues that need to be addressed by the pharmacists.

20 (43.4) Our area of focus, however, will be the inpatient pharmacy or "dispensary". Recently, the pharmacy outsourced its outpatient care to the pharmacy chain 'Rowland's' who now maintain a space next to the dispensary. In addition, they recently acquired a massive robot that took away a lot of the monotonous, tiring work like restocking shelves and pulling drugs.

16 (34.8) In order to prepare for the project, we spent a lot of time reading and familiarizing ourselves with how pharmacy education works in Scotland. After finishing classes, Scottish pharmacy students graduate and enter what is called pre-registration year. It's pretty much the same as our 4th year rotations, except it's done all at one site (wherever the student will work after licensure) and it's all coordinated through the NHS as opposed to the universities. Stage 2 vocational training is also coordinated by the NHS and is extra training for hospital pharmacists. It consists of modules, such as clinical and aseptic, and are completed by the pharmacist at their current workplace

Poland

Thailand

(1)

(n)


Health Disparities and Risks

$\begin{array}{ll}\text { My } & \text { Tourism and Sightseeing }\end{array}$

Surroundings

Customs and Culture

Architecture (Photo)

Nature (Photo)

Language and Communication

Religion and Church (Photo) History

Local People (Photo)

Current Events

Here's What
I Think and

Feel over a course of 2 years. It's been fascinating to learn about how pharmacy is different over here.

11 (23.9) A little girl, about 1 year old, has pneumonia and needs IV antibiotics and fluids. Unfortunately, there is no way for the mother to take her to the hospital because there are 5 other small children at home and no one who can take care of them. The mother is recently single and has no family in the area. The mother and child came to the clinic today with an x-ray of the lungs and abdomen. I was able to see the nodules of pneumonia that had taken root in the little girl's lungs, as well as her enlarged heart, and gas in her intestines. While medically this was an extremely interesting case, it is extremely sad because without IV antibiotics we are unsure of how well the patient will do.

44 (95.6) While in Edinburgh we did many of the typical tourist activities from climbing Arthur's Seat to touring Edinburgh Castle and walking the Royal Mile.

39 (84.8) We reviewed Thai customs and discovered a few things we were doing "wrong": When dining, you should use your fork to push food onto your spoon. The fork should never enter the mouth. Oops. It is also considered rude to point (although pointing at inanimate objects is $\mathrm{OK}$ ), and when someone bows to you in thanks (called "wei"), it is rude to not return the gesture. Oops again.

$38(82.6)$

$36(78.3)$

33 (71.7) The Term: stonker. The Meaning: something awesome, exceptional, above ordinary. Text Example: No matter the angle, the Edinburgh castle is a stonker.

$31(67.4)$

Then, we went to Iona, where we took shelter from the rain in the island's ancient and famed abbey, which was established before $600 \mathrm{AD}$ and is credited with producing the Book of Kells and popularizing the cross as a symbol of Christianity.

$23(50.0)$

20 (43.4) General admission tickets to the hill are only 5 pounds so that's where we sat. We sat on the steps and watched the Wimbledon 2012 Men's Doubles Finals, which was such a neat experience. There is also a museum that you can pay to tour, which we decided to do. I learned so much about tennis and got to see the championship cup/plate close up!

41 (89.1) Pierogies were on the menu for lunch and after a day of walking around and appreciating the scenery, we headed back into town for some amazing Lithuanian stuffed potato pancakes. This was a much appreciated meal as we have been surviving on nothing but pork, cabbage, and bread for the previous two weeks.

Transportation
Our research before the trip told us that the journey to the island would be about 3

hours and could be reached by taxi or bus. We took the sky train from our apartment to the bus station; there we took a hot second to find the booth selling tickets to Ben Phe (the town where the pier was). After boarding the bus (which looked like it was circa 1964) we were off. The bus seemed like it was missing its shocks; there was quite a bit of swaying, bouncing, and vibrating. The supposed 3-hour ride was really about 4.5 hours. The bus stopped right by the ferry pier and it was a peaceful $40-$ minute ride to the island. Before we arrived to our peaceful oasis however, we had to first get there by way of a taxi. The island taxis were trucks with benches on either
Ecuador

Scotland

Thailand

Scotland

Scotland

England

Poland

Thailand 
side of the flat bed- no seat belts, no railings, and no way would this have been allowed in the states.

Climate and Weather

Local Food and Drink (Photo)

Accommodations

Economic Differences and

Comparisons

Accommodations (Photo)

Preparation

\section{Me, Myself, Making Friends and and I Socializing}

Travel Frustrations and Inconveniences

Gratitude

Emotions
37 (80.4) Well today was the first day which the sun was shining the whole day, and apparently we are going to pay for it as it's going to rain for the next three days.

$32(69.6)$

$28(60.9)$

We are staying at the Physician's Residence at The Royal Hospital for Sick Children, so we had to stop by the hospital to pick up keys for our flat. The Residence consists of several bedrooms (each with a sink in it), 2 showers, a kitchen, a laundry room, and a library.

25 (54.3) So now we had coins like everyone else! Whoohoo! Too bad we had no idea what we could do with them. So at this point we're standing on the side of the road at a bus stop trying to read the inscriptions on British money.

14 (30.4) Interesting items on my list of things to pack:

- Toilet paper

- Malaria prophylaxis

- Ciprofloxacin for Traveler's Diarrhea

- Lots of sunscreen

- Bug spray

- Rain gear

28 (60.9) This was the farewell dinner that the pharmacy staff treated us to. It was one of the fanciest restaurants we had been to, though they have taken us to several nice places.

Each of our preceptors were there, along with the students that helped us while on our rotation and the director of pharmacy.

24 (52.3) We arrived at the [apartment] reception desk and received our keys and [our preceptor] showed us the way across campus We were very excited to get a good night's rest, but we soon found out that not only did our keys not work to our apartment, but there was also only 1 twin bed for [us] both to sleep on

22 (47.8) I can't count how many times random strangers have stopped to help us when we were lost. Though the language barrier could be an issue, they were very persistent on helping and somehow we always figured it out together.

19 (41.3) At Auschwitz, they could only cremate 500 bodies in 24 hours. At Birkenau, they could cremate 1,500 bodies in 24 hours. At these sites, nearly 1.1 million people were killed. 6 million were exterminated throughout the war. We would highly recommend this experience for everyone. Needless to say it was one of the most emotional experiences of our lives. We were fortunate enough to be able to witness the remains of one of the greatest tragedies in human history. Auschwitz is not only a humbling experience but also a reminder for every single one of us to $\mathrm{DO}$ and $\mathrm{BE}$ better for others.

16 (34.8) I just took my first dose of malaria prophylaxis (doxycycline).
Scotland

Scotland

England

Ecuador

China

England

Thailand

Poland 


\section{Issues Encountered}

Difficulty determining what to pack to stay within weight limits and facilitate regional travel

Lack of understanding of utilities and appliances

Preventative health measures

Confusion regarding utilizing

health insurance coverage

Pedestrian traffic safety

Difficulty finding accommodations or communicating with landlords or dormitory personnel on arrival

\section{Selected Supporting Quotes(s)}

I have dreaded packing for this trip for a loooooong time. Packing winter clothes for 7 weeks in a $44 \mathrm{lb}$ suitcase and a $22 \mathrm{lb}$ carry-on was NOT going to be an easy task (I can barely pack for A WEEK in Florida in under $50 \mathrm{lbs}$ ). Going internationally I can have a $50 \mathrm{lb}$ suitcase, but flying within Europe my suitcase can only be $44 \mathrm{lbs}$. Is anyone else thinking...she could pack about 4 more sweaters and a pair of shoes for that extra 6 lbs?!?! (Cause that's what I was thinking).

The hardest things right now for us are waking up on time and figuring out how to mix the scalding hot water from one faucet in the bathroom with the ice cold water from the faucet on the other side of the sink (just to give you a visual, my first attempt to wash my face was obviously to try and throw water at myself from both sinks and hope they mix before they hit my face. It didn't work. Mom suggested using the plug and letting the water mix that way. I think I look a lot cooler my way.)

After witnessing multiple patient consults involving Salmonella typhi, we have concluded gastrointestinal infections simply seem to be a way of life here. An alternate source of contamination is the water supply. It is extremely important to only use bottled water in Santo Domingo - even to bush teeth! We walked on a short trail through the woods, in which we were thankful to have received our Japanese encephalitis vaccine due to all of the standing water, to the Bayon Temple.

After dealing with my travel insurance for a day and a half (once again, felt like an eternity), I was finally able to get an appointment at 5:40PM on Wednesday evening. I had to go to a special doctor, in a way, since most here generally just see Scots who are all covered by the National Health System.

As I'm sure many of you have seen that Bangkok has been in the news for the last couple days(?). There was a bombing about a mile from where we live; we heard it on our way back from dinner but didn't know until later what had happened. It's crazy to think that we had been at the site of the first bombing several times including the night before the explosion.

In other news, [the other student] saved my life since I totally looked left today and sincerely almost got taken out by a double decker Magic Bus.

Without internet to communicate, Stephen was actually left outside in the cold (it had been snowing the day prior) locked out of the house, until the owner actually came and let him in, because we did not know he was out there.

We eventually managed to find our stop, but we couldn't figure out which train to take next. With all our luggage in tow we climbed staircase after staircase hoping to stumble upon the right train. We must have been looking pretty pathetic because the sweetest woman (who didn't even know where she

Potential Pre-Departure

Orientation Solution

Country

Scotland

Aditional communication regarding packing for extended travel and the ability to do laundry while traveling

England Remind students to ask for a thorough orientation at their accommodations, including appliances and utilities

Ecuador

Scotland required health and safety insurance coverage, including how to access it

Thailand

England Emphasize pedestrian traffic safety for APPEs in the United Kingdom

Poland Ensure each student has a clear plan for arrival that is not dependent on the ability to access the internet 
was going) offered to help us find our way, she even helped carry our bags! Seriously couldn't have done it without her.

Our apartment is located a few blocks from the station, but we quickly learned that we were not sure which direction, and the fact that night had fallen and most of the signs were only in Thai just added to our confusion. This resulted in us dragging our suitcases through the streets (and largely nonexistent sidewalks) of Bangkok which were still wet from the recent rain, retracing our steps several times.

Unprepared for flight delays

Confusion and frustration regarding public transportation

Immigration and visa issues

Scams

[The student] had just cleared security at Cincinnati airport and made it to his gate when his flight to JFK was canceled - meaning he would miss all his subsequent flights to get to Edinburgh. With 30 minutes notice, American Airlines gave him a completely new flight schedule with a completely new final destination city: GLASGOW. When given the ultimatum of either

Glasgow or no way to Scotland, he accepted. Since Glasgow is roughly an hour away from Edinburgh, he then had to figure out how to get from one city to the other (which he procrastinated until his arrival in Edinburgh).

Today was the first day that we had to navigate our way to the NHS alone. We knew which bus to get on, but did not know the direction. Luckily, we picked the right one and a 30 minute bus ride past Edinburgh Castle we arrived at our stop.

So this blog post is about two naive Americans finding their way onto a bus system for really the first time in their lives.

Just arrived in Edinburgh this morning at 7 am, only to be cordially detained at customs. All was fixed thanks to our wonderful preceptor but apparently you should be very, veeeery careful what words you choose to say. A little more stringent than we were prepared for.

He rushed us into the cab, told us every place we wanted to go was closed and that there were traffic jams everywhere, and ended up dropping us off in the middle of no where and charging us 5 times what the actual value of the trip was worth. We knew it was happening the whole time as we had learned of this being a common occurrence, but did not know what to do in the heat of the moment. We definitely learned our lesson and are more aggressive now when finding taxis.

Unfamiliar with culturally based greetings, dress norms, table etiquette, and tipping practices
When arriving anywhere you must greet everyone in the room with a hug and kiss on the cheek (even if you saw the person less than 24 hours ago). Also, you must also say good-bye with a hug and a kiss on the check every time you leave.

We learned the night before from the Irish people that tipping here is apparently not always done. They find the American culture of always having to tip strange.

It is common for people to use spoons AND a fork. You will never see someone eat with a fork alone because it is bad manners to eat directly off of your fork. Instead, it is used to scoop food onto your spoon, from which you will then eat off of.
Thailand

Scotland Provide general guidance on how to work with airline personnel to reschedule flights and what accommodations and compensation are required for delays and cancellations

Scotland

Country-/city-specific information to be provided during pre-travel orientation

England

Scotland

Provide additional training and discussion

Thailand

Add follow-up discussion of countryspecific US Department of State resource or send the resource to students prior to their pre-departure orientation in order to have more specific discussions

Ecuador Incorporate these practices into pre-travel orientation
England

Thailand 
Although we felt we had dressed appropriately according to what we understood the dress code to be (covered toes, covered shoulders, covered

knees) we were not appropriate enough to enter the Grand Palace and so had to rent sarongs for a 200 baht deposit apiece

Did you know that you have to PAY to use the bathrooms there!?!? I also stood staring at the barriers to the bathroom with a confused look on my face before I realized that they really weren't joking. Fair or not, my need to use the bathroom after a 2 hour train ride and an entire bottle of water was necessary, so I coughed up my 30 pence.

Language barriers

Once we arrived to our destination we realized just how challenging it can be when it comes to the language barrier. This became quite comical when we tried to communicate to our cab driver where we needed to go, when checking in with our doorman upon arrival, and again at the restaurant where we had dinner. I think we are going to have to "you-tube" some common Polish phrases to get us through the next couple of days! I don't know how to say "vegetarian" or "no meat", but I had one of the pharmacists we work with write it on a piece of paper to show while ordering and that has helped.
Thailand

England

Poland

Requesting students learn a few basic phrases in the language of their host country and other countries where they plan to visit Carry allergy and dietary information written in the local language

Thailand 
Figure 1. Number of Codes Isolated Per Category in Each Country.

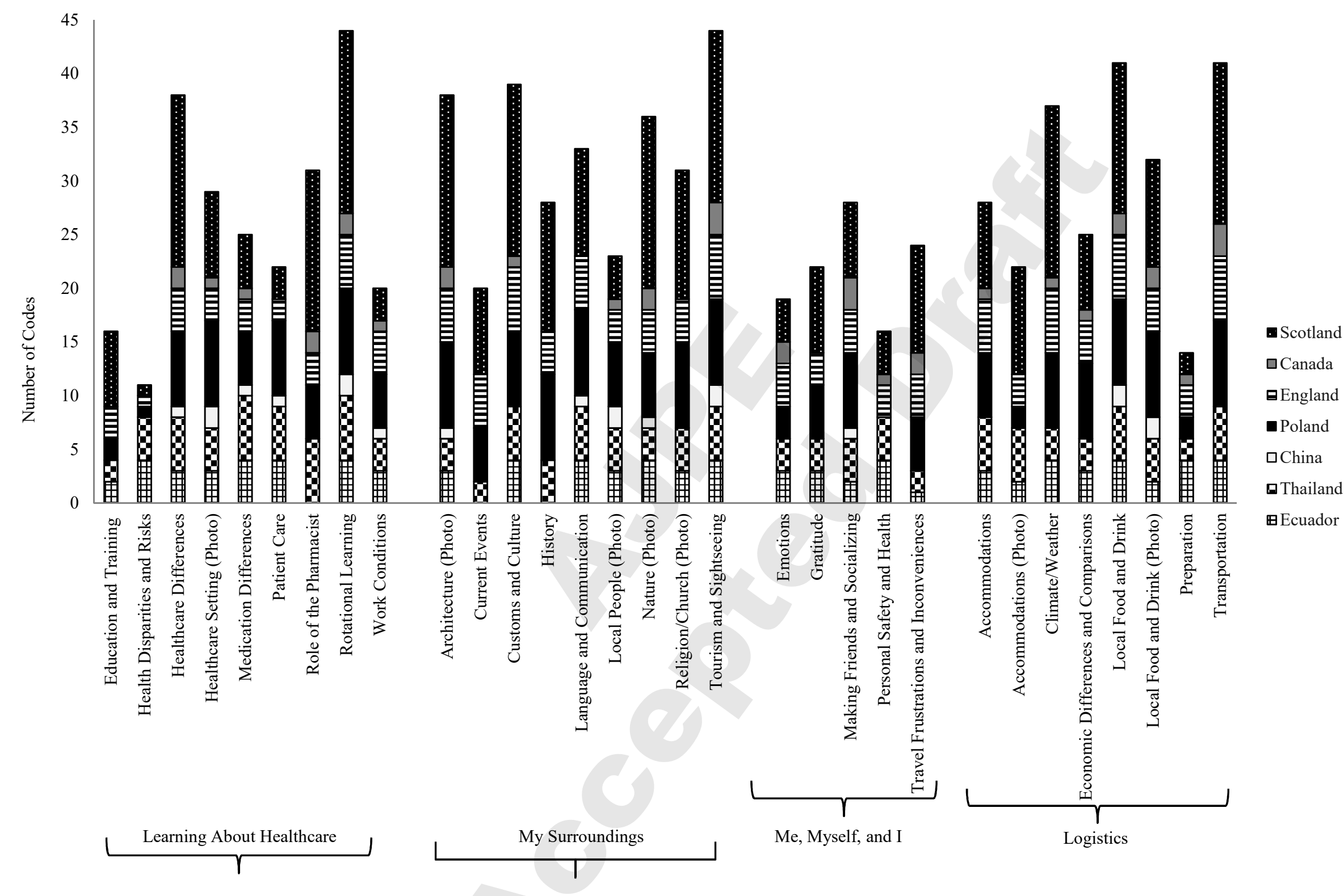

